\title{
¿Travestismo o transexualidad? Divergencia en la cobertura mediática del asesinato de un hombre travestido
}

\author{
Trabestismoa edo transexualitatea? \\ Dibergentzia trabestitutako gizon baten hilketaren estaldura mediatikoan \\ Cross-dressing or transsexualism? \\ Divergence in media coverage of the murder of a transvestite man
}

\author{
Rubén Olveira-Araujo $\star$ \\ Universidad del País Vasco/Euskal Herriko Unibertsitatea (UPV/EHU)
}

\begin{abstract}
RESUMEN: Las representaciones mediáticas sobre las identidades trans(género) se han multiplicado en los últimos años. Para contribuir a la escasa literatura académica sobre travestismo en el área de Comunicación, se ha examinado mediante un análisis de contenido la cobertura mediática del asesinato de un hombre travestido en Castilla y León. Los resultados indican que la prensa continúa mezclando diferentes hechos de diversidad sexual. Sin embargo, se observa un cambio en la direccionalidad de dicha confusión: de confundir la transexualidad con el travestismo, ahora parece que se confunde el travestismo con la transexualidad. Pese a todo, también sugieren que la cobertura mediática sobre la diversidad sexual ha mejorado.
\end{abstract}

PALABRAS CLAVE: Transgénero; LGTB; Periodismo; Representación mediática; Travestismo; Transexualidad.

ABSTRACT: Media portrayal of trans(gender) identities have multiplied in recent years. In order to contribute to the scarce academic literature on transvestism in the area of Communication, the media coverage of the murder of a transvestite man in Castilla y León has been examined through a content analysis. The results indicate that the press continues mixing different facts of sexual diversity. Moreover, from confusing transsexualism with cross-dressing, now it seems that cross-dressing is confused with transsexualism. Despite all this, they also suggest that media coverage of sexual diversity has improved.

KEYWORDS: Transgender; LGBT; Journalism; Media Representation; Cross-dressing; Transsexualism.

\footnotetext{
* Correspondencia a / Corresponding author: Rubén Olveira-Araujo. Facultad de Ciencias Sociales y de la Comunicación de la UPV/EHU. Campus de Bizkaia. Barrio Sarriena, s/n (48940 Leioa-Bizkaia) - Ruben.olveira@ehu.eus - https://orcid.org/0000-0003-4797-8746

Cómo citar / How to cite: Olveira-Araujo, Rubén (2021). "¿Travestismo o transexualidad? Divergencia en la cobertura mediática del asesinato de un hombre travestidon, Zer, 26(51), 121-137. (https://doi.org/10.1387/zer.22550).

Recibido: 22 febrero, 2021; aceptado: 25 junio, 2021.

ISSN 1137-1102 - elSSN 1989-631X / (C) 2021 UPV/EHU

(c) (i) $\odot$ Esta obra está bajo una Licencia

By No Creative Commons Atribución-NoComercial-SinDerivadas 4.0 Internacional
} 


\section{Introducción}

La diversidad sexual es noticia. Las representaciones mediáticas sobre estas realidades se han multiplicado en los últimos años, tendencia que a nivel internacional parece especialmente notoria en lo que a las identidades trans(género) se refiere (Âkerlund, 2018; Billard, 2016). Este incremento resulta relevante dado que, según la teoría de la agenda setting (McCombs, 2006), los medios de comunicación han demostrado ser capaces de moldear a largo plazo la opinión pública mediante la cualidad acumulativa de sus efectos cognitivos. De esta manera, están relacionados con las políticas públicas sobre las estrategias de gestión de la diversidad sexual. Además, los news media se han alzado tradicionalmente como un canal crucial para dar a conocer estas realidades, una labor de visibilización y sensibilización fundamental que a día de hoy todavía mantienen y a través de la que pueden educar y promocionar la diversidad sexual al tiempo que prevenir y combatir los discursos del odio.

Fomentar imágenes positivas y combatir los discursos del odio que sustentan el prejuicio negativo al que se enfrentan las personas trans(género) - la transfobia- es una responsabilidad deontológica. Las consecuencias más graves de esta discriminación negativa están relacionadas con una mayor mortalidad de quienes la padecen, aumentando el riesgo de ser víctima de asesinato (Transgender Europe, 2018) y de suicidio (Grant, Mottet, Tanis, Harrison, Herman y Keisling, 2011). Sin embargo, la transfobia va más allá e incrementa considerablemente la probabilidad de sufrir acoso escolar y laboral, violencia fisica, abandono escolar, vivir en pobreza extrema, etc.

Pese a las notorias diferencias entre los hechos de diversidad sexual que se integran dentro del concepto trans(género), gran parte de la literatura en Comunicación, principalmente aquella de carácter anglosajón, ha optado por su análisis genérico en los mensajes periodísticos (por ejemplo, Barker-Plummer, 2013; Billard, 2016, Capuzza, 2016). En este artículo, en cambio, se prefiere diferenciar entre las manifestaciones de diversidad sexual que integra dicho concepto. De hecho, de cara a este análisis resulta imprescindible distinguir al menos entre dos de ellos: la transexualidad y el travestismo.

Si bien existen otras miradas posibles, como la perspectiva queer (Butler, 2004), desde un enfoque sexológico (Landarroitajauregi, 2000) la transexualidad se trata de una situación de tránsito - social pero también personal - en la forma de vivir y expresar la sexualidad, comprendida esta última como la manera de vivir(se) como el hombre o la mujer que se es. Esta es generada por una discordancia entre la clasificación sexual propia (autosexación) y la ajena (alosexación), a la que en Sexología se denomina como transexación. Por otra parte, el travestismo es la práctica de vestirse con ropas social y culturalmente consideradas del sexo complementario. En cuanto 
a trangénero y su diminutivo trans, suponen unos conceptos paraguas que abarcan, además de la transexualidad y el travestismo, otros hechos de diversidad sexual relacionados con la identidad sexual y su expresión.

La literatura académica en el Estado español sobre la cobertura mediática de la transexualidad es escasa y del travestismo, inexistente. Una de estas aproximaciones es la de Carratalá (2011), quien identifica en la prensa diaria valenciana el incorrecto nombramiento y clasificación sexual de una mujer marroquí en situación de transexualidad asesinada en 2009, a la que se confunde con un hombre travestido. Este estudio destaca la indefinición de la identidad sexual de la víctima al intercambiar indistintamente el género gramatical, el uso mayoritario de fuentes institucionales y una representación general entre la criminalización y la culpabilización de la víctima. Velasco Malagón (2017), por otro lado, analiza la representación de la transexualidad en general durante 2013 en los diarios El País, El Mundo y ABC y constata una confusión similar a la de los periodistas de otros territorios a la hora nombrar adecuadamente algunas de las dimensiones de este hecho de diversidad sexual.

Marini, Medina y Rodrigo (2010) investigaron la cobertura mediática del embarazo de Thomas Beatie, un hombre estadounidense en situación de transexualidad, en once cibermedios de cuatro países. De este análisis concluyeron que los medios no van más allá del hecho noticioso, obviando la reflexión social sobre la transexualidad. También que en la cobertura mediática de estas realidades se recurre principalmente a fuentes expertas. En cuanto a Olveira-Araujo (2019), analiza la representación de la transexualidad durante 2017 en los cibermedios El País, El Mundo, Play Ground y Vice adaptando los indicadores propuestos por Billard (2016). De esta manera, halla desde conceptualizaciones incorrectas en la representación de la transexualidad a el uso inadecuado de la terminología asociada, entre otros.

Los resultados de ambos trabajos coinciden con los obtenidos en otras investigaciones a nivel internacional. Por ejemplo, Billard (2016) comprueba que las formas de «deslegitimación» menos frecuentes son aquellas más explícitas, como la difamación y los insultos, mientras que aquellas más implícitas, como el incorrecto nombramiento y clasificación sexual o la confusión entre diferentes hechos de diversidad sexual, son más habituales. Respecto a la cobertura de sucesos, Chávez (2010) señala la criminalización y culpabilización de las víctimas transgénero. Además de esto último, Barker-Plummer (2013) añade el uso frecuente de fuentes institucionales y la importancia de recurrir a fuentes del tercer sector para una cobertura más completa y adecuada. Baptista y Himmel (2016) constatan tanto el uso de ambos géneros gramaticales para referirse a las personas transgénero como la utilización indistinta de los términos travesti y transexual. Por otro lado, Leandro y Caldas-Coulthard (2018) observan que el travestismo se señala como un factor incriminante, mientras la transexualidad no posee tal desprestigio. 
Con el objetivo principal de contribuir al conocimiento sobre la representación mediática del travestismo en el Estado español, el presente trabajo ha optado por realizar un estudio de caso (Yin, 2009). Este tipo de aproximaciones exploratorias permiten abordar el objeto de estudio con profundidad y, así, ofrecer nuevas preguntas e hipótesis a comprobar en futuras investigaciones.

A la hora de elegir el caso de análisis se tuvo en cuenta que, a pesar de que las noticias sobre sucesos suponen una pequeña porción de la representación mediática de las identidades trans(género), estos hechos noticiosos presentan la mayor cantidad de «elementos deslegitimadores» (Billard, 2016). De hecho, una parte considerable de los estudios de caso que abordan estas realidades se centran en ellos. Asimismo, la cobertura mediática de sucesos es más habitual en la prensa local que en la nacional (MacKenzie y Marcel, 2009). Por esa razón se ha seleccionado para el análisis la cobertura mediática del caso de asesinato de La Eli en la prensa distribuida en Castilla y León. Esta elección permite también analizar el cumplimiento de los criterios deontológicos relacionados con víctima. Los hechos son los siguientes:

En la madrugada del 12 de agosto de 2018 Francisco Javier (F.J.), un hombre travestido de 59 años, al que apodaban la Eli, fue hallado inconsciente con claros indicios de haber sufrido una agresión a la entrada del centro comercial Vallsur (Valladolid). Poco después de ingresar en el hospital entró en estado de coma y falleció el 22 de septiembre. En cuanto al autor del delito de asesinato, un menor de 15 años, se entregó un día después de la agresión. El Juzgado de Menores dictó sentencia el 30 de abril de 2019.

Para el análisis de este caso, se han planteado las siguientes preguntas de investigación:

— RQ1. ¿Cómo se ha representado el travestismo?

- RQ2. ¿Cuáles han sido las fuentes consultadas?

— RQ3. ¿Se cumple con los criterios deontológicos pertinentes?

\section{Método}

Para responder a las preguntas de esta investigación, este trabajo ha optado por un análisis de contenido cuantitativo (Krippendorff, 2004). Esta técnica es capaz de extraer los componentes, relaciones internas y transformaciones de la cobertura mediática y lograr inferencias reproducibles y válidas.

El corpus de análisis está conformado por los artículos publicados en la prensa impresa generalista que se distribuye en el territorio de Castilla y León sobre el caso 
de la Eli $(n=31)$. Tras una búsqueda exhaustiva, los diarios que se hicieron eco de este suceso fueron el Diario de León, El Diario de Valladolid (edición local El Mundo), El Norte de Castilla, La Opinión-El Correo de Zamora, El Mundo, ABC y La Razón. Las cuatro primeras cabeceras son de carácter local, mientras las tres siguientes de ámbito estatal.

La selección de los artículos se realizó a través del servicio de hemeroteca de MyNews, diferenciándose dos fases. En la primera, que comprende la cobertura mediática desde la agresión a la muerte de F.J., se acotó el periodo de búsqueda del 12 de agosto 2018 al 15 de octubre 2018 y se utilizó la siguiente expresión booleana: Eli OR Vallsur OR «Avenida de Zamora». De los 289 resultados iniciales, tras desestimar aquellos que no tenían que ver con el estudio de caso y eliminar los duplicados, se seleccionaron 22 noticias. En la segunda, que se extiende desde el seguimiento del caso hasta el desenlace judicial, el periodo de búsqueda se acotó del 16 de octubre de 2018 al 31 de mayo de 2019 y la expresión booleana se restringió a Eli AND Vallsur. Tras efectuar el mismo filtrado que en la primera fase con los 25 artículos resultantes, se seleccionaron 9 informaciones adicionales.

La fase de codificación, que fue asistida por el software NVivo, se realizó en base a catorce variables agrupadas en cinco dimensiones: datos generales, forma, origen del mensaje, diversidad sexual e indicadores deontológicos (tabla I). En ella se incluyen instrumentos como la clasificación de los géneros periodísticos de Gomis (1991); una adaptación de la Escala de Atención de Richard Budd (1964), que sirve para cuantificar la preeminencia que otorga cada medio a los diferentes hechos noticiosos publicados; o los indicadores deontológicos extraídos de diferentes documentos $^{1}$. Para comprobar su adecuación al estudio de caso se realizó un análisis cualitativo preliminar. Asimismo, para garantizar la fiabilidad de los resultados se llevó a cabo un repaso exhaustivo de la codificación un mes después.

El análisis se realizó a través del software SPSS. Se emplearon estadísticos descriptivos básicos y, con el fin de comprobar estadísticamente la existencia de dos líneas de representación diferenciables, se recurrió a un análisis de conglomerados en dos fases.

\footnotetext{
1 A la hora de seleccionar los indicadores deontológicos se tuvieron en cuenta el Código internacional de ética periodística de la Unesco (1983), el Código deontológico europeo de la profesión periodística (Parlamento de Europa, 1993), la Declaración de principios de la FIP sobre la conducta de los periodistas (FIP, 1986), el Código deontológico de la FAPE (2017) y la Declaración de principios de la profesión periodística en Catalunya (Colegio de Periodistas de Cataluña, 2016), por un lado, y el Libro de estilo de El Mundo (De la Serna, 1996), el Libro de estilo de ABC (Vigara Tauste, 2001) y el Libro de estilo de Vocento (De Sousa, 2003), por otro. También se valoraron las Recomendaciones sobre el tratamiento de las personas lesbianas, gays, bisexuales, transgéneros e intersexuales (LGBTI) en los medios audiovisuales del Consejo del Audiovisual de Cataluña (2017) y las pautas sugeridas por Carratalá (2011).
} 
TABLA I

Plantilla de Codificación

\begin{tabular}{|c|c|c|}
\hline Grupos & Variable & Categorías \\
\hline \multirow{2}{*}{$\begin{array}{c}\text { Datos } \\
\text { generales }\end{array}$} & Cabecera & - \\
\hline & Periodo & Agresión y Detención, Muerte y Luto, Juicio. \\
\hline \multirow[b]{2}{*}{ Forma } & Género periodístico & $\begin{array}{l}\text { Noticia, Reportaje, Entrevista, Crónica, Crítica, Co- } \\
\text { mentario. }\end{array}$ \\
\hline & Preeminencia & $\begin{array}{l}\text { Se añade un punto por cada supuesto cumplido: 1) si } \\
\text { el titular va a tres columnas; 2) si la noticia está ubi- } \\
\text { cada en la mitad superior de la página; 3) si está en } \\
\text { página impar; 4) si aparece en portada; 5) si además } \\
\text { abre la misma; y 6) si cuenta con ilustración fotográ- } \\
\text { fica o gráfica. }\end{array}$ \\
\hline \multirow{3}{*}{$\begin{array}{l}\text { Origen } \\
\text { del mensaje }\end{array}$} & Firma & Autor, Medio, Agencias. \\
\hline & Tipo de Fuente & \multirow{2}{*}{ Gubernamental, Activista, No Oficial. } \\
\hline & Extensión de Fuentes & \\
\hline \multirow{3}{*}{$\begin{array}{l}\text { Diversidad } \\
\text { sexual }\end{array}$} & Sexo & Hombre, Mujer, Ambiguo, No especificado. \\
\hline & Hecho de Diversidad Sexual & $\begin{array}{l}\text { Transexualidad, Travestismo, Transgenerismo, Ho- } \\
\text { mosexualidad, No especificado. }\end{array}$ \\
\hline & Términos de identificación & - \\
\hline \multirow{4}{*}{$\begin{array}{l}\text { Indicadores } \\
\text { deontológicos }\end{array}$} & Identificación & \multirow{4}{*}{ Sí, no. } \\
\hline & Respeto a la intimidad & \\
\hline & Criminalización /Culpabilización & \\
\hline & Sustantivizar hecho de diversidad & \\
\hline
\end{tabular}

Fuente: elaboración propia.

\section{Resultados}

\subsection{Datos formales}

En la prensa impresa generalista de Castilla y León se publicaron 31 artículos sobre el caso de $\mathrm{La}$ Eli, obteniendo una puntuación directa de 88 en la adaptación de la Escala de Atención de Budd y una media de 2,8 puntos $(S D=1,6)$. De los tres periodos analizados (figura 1), el primero, Agresión y Detención, es el que alcanzó mayor visibilidad mediática $(n=13, p d=41, M=3,2, S D=2,1)$. Los dos restantes obtuvieron una repercusión similar: si bien la cobertura de la fase Juicio $(n=9$, 
$p d=25, M=2,4, S D=1,1)$ logró resultados más elevados que el periodo de Muerte $y$ Luto $(n=9, p d=22, M=2,8, S D=1)$, hay que tener en cuenta que la segunda fue cubierta por tres cabeceras adicionales.

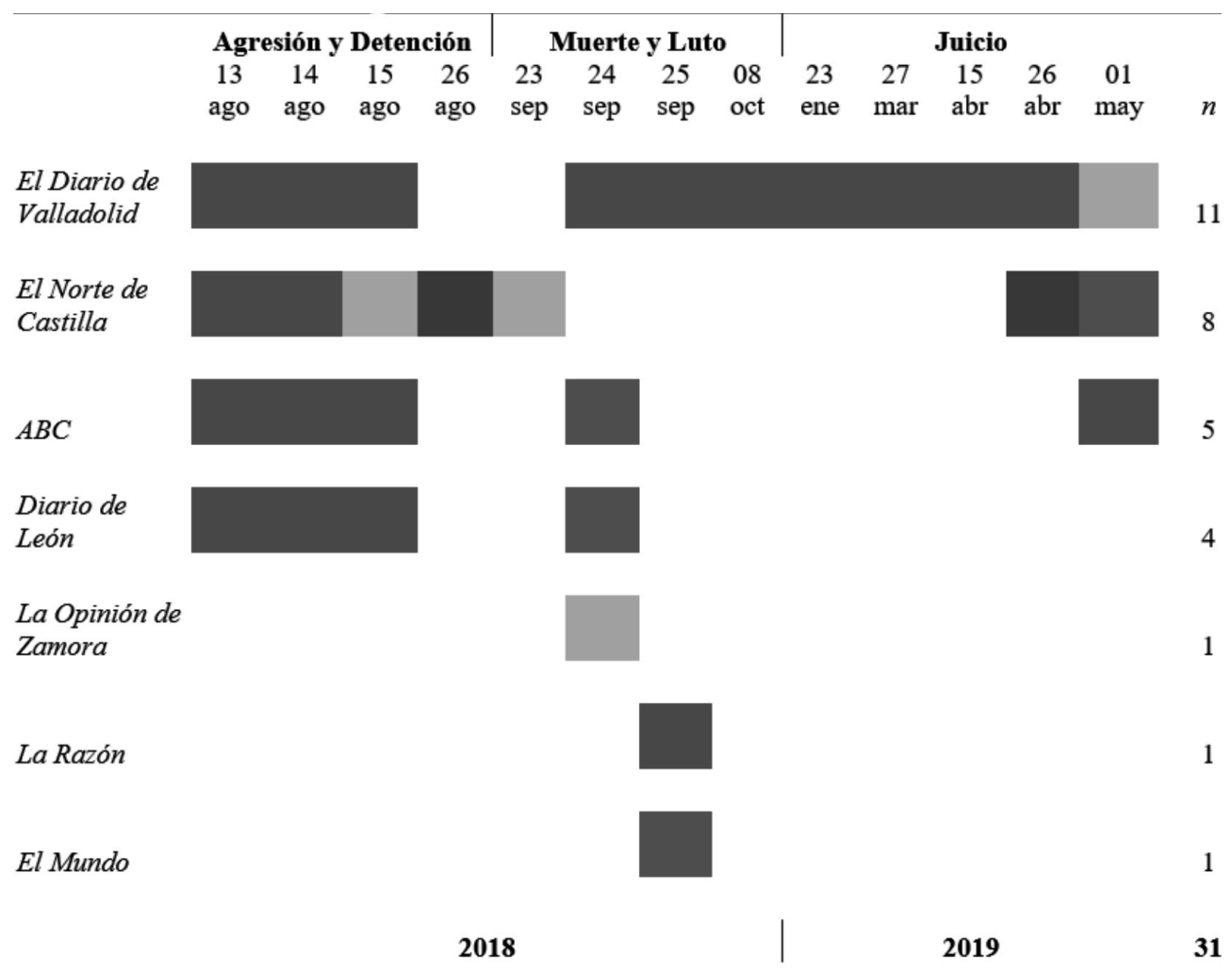

\section{Hombre}

FIgURA 1

Evolución de la identidad sexual

Salvo un artículo de opinión y un reportaje en forma de obituario, el resto de las piezas recurrieron al género de noticia o información $(n=29,94 \%)$. Respecto a las cabeceras, además de ofrecer un seguimiento continuado, El Diario de Valladolid $(n=11, p d=42, M=3,8, S D=1,3)$ fue la que más importancia otorgó al caso de La Eli. Le siguió El Norte de Castilla $(n=8, p d=26, M=3,2, S D=1,5)$, que ofreció un mayor seguimiento en el primer periodo e inició el segundo con su primicia; $A B C(n=5, p d=7, M=1,4 S D=0,5)$; y el Diario de León $(n=4, p d=6$, 
$M=1,5, S D=1,3)$. Las tres cabeceras restantes solamente publicaron un artículo para dar noticia del fallecimiento.

\subsection{ORIGEN DEL MENSAJE}

Más de la mitad de los artículos fueron firmados por redactores $(n=17,55 \%)$, un tercio se atribuyó a agencias $(n=11,35 \%)$ y los restantes al medio en general $(n=3,10 \%)$. El Diario de Valladolid, El Norte de Castilla y La Razón recurrieron prácticamente en su totalidad a redactores propios, mientras que en $A B C$, el Diario de León y La Opinión primaron las noticias de agencia. Esta distinción por cabeceras coincide con las diferencias entre periodos: en la fase en la que estos últimos diarios estuvieron ausentes (Juicio) la totalidad de las firmas correspondieron al medio (redactores concretos y medio en general), mientras que cuando estuvieron presentes (Agresión y Detención y Muerte y Luto) estas bajaron a la mitad. Asimismo, los artículos que puntuaron más alto en la escala de atención tendían a ir firmados por redactores, mientras que aquellos que puntuaron más bajo se atribuyeron a agencias.

Cerca de la mitad de los artículos recurrieron a fuentes institucionales $(n=15$, 48\%), como la Policía Municipal y Nacional, mientras la otra mitad combinó fuentes tanto institucionales como activistas $(n=15,48 \%)$; solo un artículo usó únicamente fuentes activistas. En aquellos artículos en los que se recurrió a ambas fuentes, la extensión del texto que provenía de fuentes institucionales suponía el 76\%; el 24\% restante se atribuyó a fuentes del activismo, entre las que destacaron la Fundación Triángulo y, en menor medida, ATC Raimbow. Esta última distinción es especialmente importante dado que mientras la primera organización abogó por referirse a F.J. como una mujer en situación de transexualidad, la segunda declaró que se trataba de un hombre gay que practicaba el travestismo.

Por periodos, durante la fase de Agresión y Detención los artículos se repartieron casi a partes iguales entre el uso de fuentes institucionales y el uso combinado de fuentes tanto institucionales como activistas. En el periodo de Muerte y Luto, en cambio, prácticamente la totalidad combinaron ambas fuentes y uno recurrió solo a fuentes activistas, mientras que durante la cobertura mediática del Juicio la tendencia se invirtió: se utilizaron casi de forma exclusiva fuentes institucionales. Esta distinción por periodos coincide también con las diferencias entre cabeceras. No se observaron diferencias en función de la firma.

\subsection{IDENTIDAD SEXUAL}

J.F. fue representado habitualmente como hombre, si bien se observó una evolución entre los distintos periodos. Durante la fase de Agresión y Detención este 
porcentaje ascendió a casi la totalidad (tabla II). Sin embargo, durante las dos siguientes este bajó a la mitad a favor de considerarlo mujer, no especificar el sexo o intercambiar el sexo durante el texto a través del género gramatical. En cuanto a las cuatro cabeceras que hicieron mayor seguimiento del caso, se observaron dos líneas (figura 1): mientras que El Diario de Valladolid le categorizó como hombre, El Norte de Castilla, al igual que $A B C$ y el Diario de León, comenzó representándolo como hombre, pero en las fases posteriores intercambió mujer, sexo ambiguo y no especificar.

TABLA II

Sexo por periodo

\begin{tabular}{l|rr|rr|rr|rr|r}
\hline & \multicolumn{2}{|c|}{ Hombre } & \multicolumn{2}{c|}{ Mujer } & \multicolumn{2}{c|}{ Ambiguo } & \multicolumn{2}{c|}{ No especificado } & Total \\
\cline { 2 - 11 } & \multicolumn{1}{|c|}{$\mathbf{n}$} & $\%$ & $\mathbf{n}$ & $\%$ & $\mathbf{n}$ & $\%$ & $\mathbf{n}$ & $\%$ & $\mathbf{n}$ \\
\hline Agresión y detención & 12 & $92 \%$ & 0 & $0 \%$ & 0 & $0 \%$ & 1 & $8 \%$ & 13 \\
Muerte y luto & 4 & $44 \%$ & 2 & $22 \%$ & 0 & $0 \%$ & 3 & $33 \%$ & 9 \\
Juicio & 5 & $56 \%$ & 1 & $11 \%$ & 2 & $22 \%$ & 1 & $11 \%$ & 9 \\
\hline Total & 21 & $68 \%$ & 3 & $10 \%$ & 2 & $6 \%$ & 5 & $16 \%$ & 31 \\
\hline
\end{tabular}

Fuente: elaboración propia.

\subsection{HECHO DE DIVERSIDAD SEXUAL}

El hecho de diversidad sexual dominante para representar a J.F. fue la transexualidad $(n=10,32 \%)$, seguido del travestismo $(n=7,23 \%)$, tanto utilizando dicho término como a través de la expresión «hombre vestido de mujer». Aun así, un tercio no aludieron a ningún hecho de diversidad sexual $(n=10,32 \%)$. Al igual que por sexo, también se observaron diferencias entre periodos y cabeceras (figura 1). Por un lado, mientras que en la fase de Agresión y Detención se optó principalmente por calificarle como travesti o no especificar, en la de Muerte y Luto más de la mitad de los artículos le categorizaron como transexual, porcentaje que se redujo en la de Juicio a favor de no especificar ningún hecho de diversidad sexual. Por otro, El Diario de Valladolid apenas especificó y cuando lo hizo le categorizó como transgénero en el periodo de Agresión y Detención y como homosexual en la fase de Muerte y Luto. El Norte de Castilla, en cambio, recurrió sobre todo a transexual en el segundo y tercer periodo y a travesti en el primero, siendo la misma línea que siguió el $A B C$. El Diario de León, por otra parte, durante Agresión y Detención no especificó, pero en Muerte y Luto también se pasó a la transexualidad. 


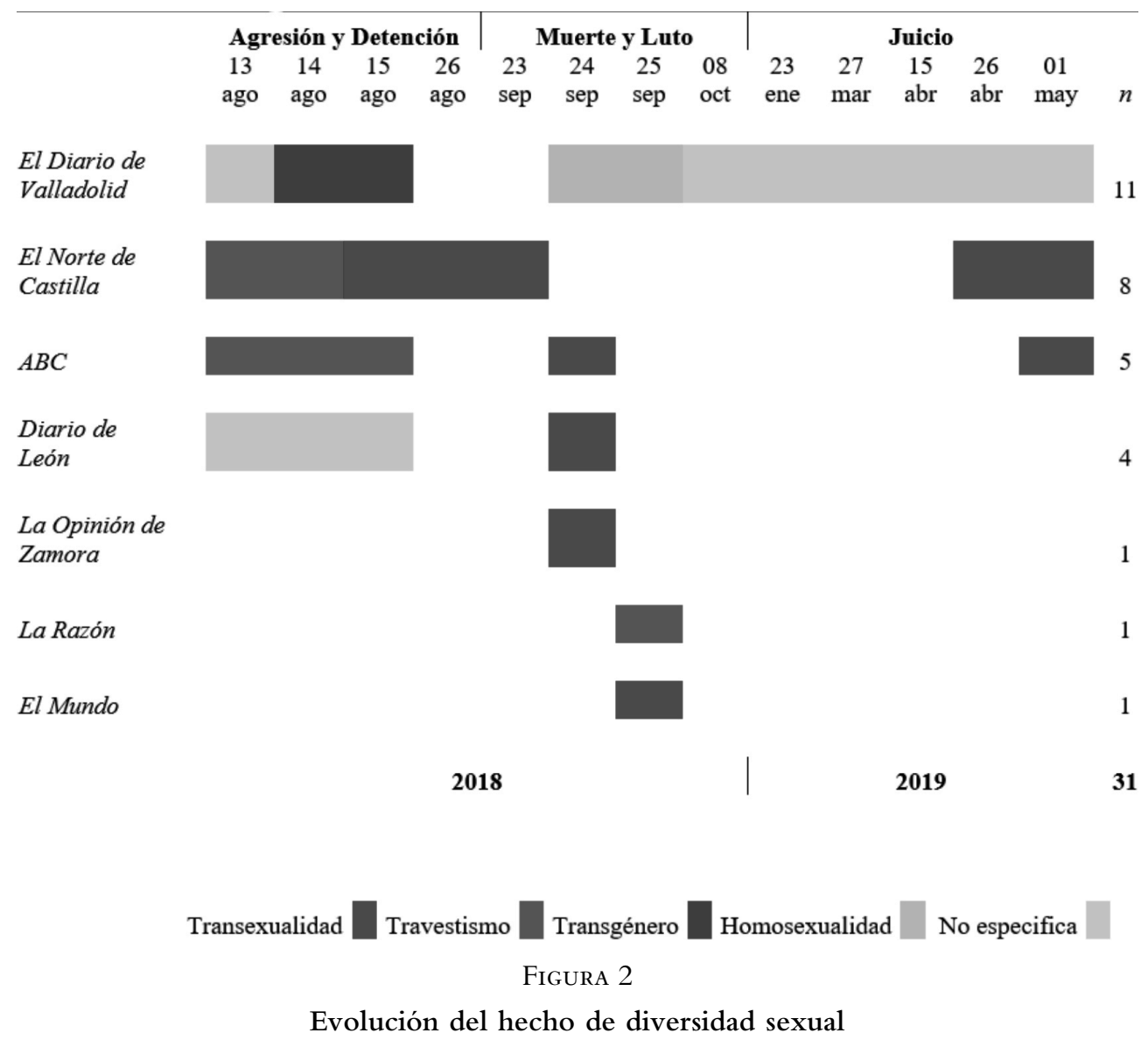

Combinando las variables Cabecera, Periodo, Sexo y Hecho de Diversidad (esta última recodificada en dos categorías: Transexualidad y Travestismo y otros), el análisis de conglomerados bietápico confirmó la existencia de dos líneas de representación. La agrupación de artículos más numerosa $(n=20,65 \%)$, que fue cuantitativamente liderada por El Diario de Valladolid, abarcó casi todas las informaciones del primer periodo, reduciéndose a la mitad en los dos siguientes, y presentó a F.J. como hombre tranvestido, homosexual o transgénero cuando se especificó; es decir, se refirieron a dimensiones que efectivamente interseccionaban la vida F.J. El segundo grupo $(n=11,35 \%)$, liderado por El Norte de Castilla, adquirió mayor relevancia en el segundo y tercer periodo y representó erróneamente a F.J. como una persona en situación de transexualidad. Sin embargo, los artículos de esta línea no se pusieron de acuerdo respecto a su identidad sexual, cambiando indiscriminadamente su clasificación sexual —incluso dentro del mismo artículo-. 
Los términos más empleados para referirse a J.F. (tabla III) corroboraron estos resultados: hombre se utilizó en mayor medida que mujer y durante el primer periodo se usaron más travesti y palabras relacionadas con su vestimenta, como vestido y disfrazado, mientras que transexual tomó su relevo en el segundo. Aun así, el término más empleado fue víctima. Este descendió en la fase de Muerte y Luto a favor de identificarle (Eli, Javier, Francisco) para aumentar en el periodo del Juicio. Hay que destacar que el apodo Eli se utilizó con mayor frecuencia que Javier. Asimismo, es reseñable la frecuencia de años, que deja constancia de la atención que presta la cobertura mediática a la diferencia de edad entre los protagonistas del caso.

TABLA III

Términos de identificación

\begin{tabular}{|c|c|c|c|c|c|c|c|c|c|}
\hline \multicolumn{2}{|r|}{ Términos } & \multicolumn{6}{|c|}{ Periodo } & \multirow{2}{*}{\multicolumn{2}{|c|}{ Total }} \\
\hline Palabra & Palabras similares & \multicolumn{2}{|c|}{$\begin{array}{c}\text { Agresión } \\
\text { y detención }\end{array}$} & \multicolumn{2}{|c|}{$\begin{array}{c}\text { Muerte } \\
\text { y luto }\end{array}$} & \multicolumn{2}{|c|}{ Juicio } & & \\
\hline víctima & víctima, víctimas & 39 & $35 \%$ & 22 & $19 \%$ & 52 & $46 \%$ & 113 & $21 \%$ \\
\hline eli & Eli, Ely & 0 & $0 \%$ & 27 & $37 \%$ & 46 & $63 \%$ & 73 & $14 \%$ \\
\hline años & años & 26 & $65 \%$ & 9 & $23 \%$ & 5 & $13 \%$ & 40 & $8 \%$ \\
\hline hombre & hombre & 36 & $95 \%$ & 2 & $5 \%$ & 0 & $0 \%$ & 38 & $7 \%$ \\
\hline persona & persona, personas & 17 & $53 \%$ & 15 & $47 \%$ & 0 & $0 \%$ & 32 & $6 \%$ \\
\hline transexual & transexual & 3 & $13 \%$ & 16 & $67 \%$ & 5 & $21 \%$ & 24 & $5 \%$ \\
\hline mujer & mujer & 18 & $90 \%$ & 1 & $5 \%$ & 1 & $5 \%$ & 20 & $4 \%$ \\
\hline javier & javi, javier & 0 & $0 \%$ & 7 & $50 \%$ & 7 & $50 \%$ & 14 & $3 \%$ \\
\hline vestido & vestía, vestido & 10 & $83 \%$ & 1 & $8 \%$ & 1 & $8 \%$ & 12 & $2 \%$ \\
\hline travesti & travesti, travestida, travestido & 7 & $78 \%$ & 1 & $11 \%$ & 1 & $11 \%$ & 9 & $2 \%$ \\
\hline agredido & agredida, agredido & 7 & $88 \%$ & 1 & $13 \%$ & 0 & $0 \%$ & 8 & $2 \%$ \\
\hline francisco & francisco & 0 & $0 \%$ & 2 & $25 \%$ & 6 & $75 \%$ & 8 & $2 \%$ \\
\hline disfrazado & disfrazado & 6 & $100 \%$ & 0 & $0 \%$ & 0 & $0 \%$ & 6 & $1 \%$ \\
\hline & & & & & & & $\mathrm{n}$ & 397 & $75 \%$ \\
\hline & & & & & & & $\mathrm{N}$ & 528 & $100 \%$ \\
\hline
\end{tabular}

Fuente: elaboración propia.

\subsection{INDICADORES DEONTOLÓGICOS}

La mayoría de los artículos cumplieron los indicadores considerados $(n=21$, $58 \%)$. Dos quintas partes de las informaciones identificaron a J.F. $(n=13,42 \%)$, ya sea por su nombre o por su apodo. Sin embargo, no se consideraron como falta debido a que todas las identificaciones fueron tras su muerte, una vez que la asociación ATC Rainbow hiciera público su nombre. Por otra parte, cerca de un tercio de los artículos se refirieron a los hechos de diversidad de forma sustantiva $(n=9,29 \%)$ 
(por ejemplo, «Muere una transexual apaleada por un menor de 15 años en Valladolid»). Un artículo culpabilizó a J.F. mediante la repetida alusión en que iba «disfrazado de mujer» $(n=4)$ y otro como intromisión gratuita en la intimidad por la expresión «era muy conocida en los ambientes de ocio nocturno vallisoletano». En ambos casos se trató de autores que no hicieron seguimiento del caso.

\subsection{ReCONSTRUCCión DEL SEGUimiento MEdiÁtico}

Combinando los datos presentados hasta el momento se puede extraer que durante el periodo inicial de Agresión y Detención se representó a J.F. como hombre y en algunos artículos se señaló que practicaba el travestismo en el momento de la agresión. La excepción la supone la información publicada por El Norte de Castilla el 15 de agosto (figura 1), que pasó a categorizarle como transexual — sin especificar su sexo- como recomendación de la Fundación Triángulo, de la cual se hizo eco en el texto. Desde ese momento, El Norte de Castilla calificó a J.F. como transexual en todas sus informaciones y, en cuanto al sexo, se debatió entre mujer y la ambigüedad.

La fase mediática de Muerte y Luto se inició con la primicia de este mismo diario, que fue su única pieza durante este periodo. Guiados por la caracterización de este artículo, en esta segunda fase $A B C$, el Diario de León y El Mundo representaron a J.F. como una mujer en situación de transexualidad y La Opinión de Zamora como una persona en situación de transexualidad, sin especificar su sexo. El Diario de Valladolid, en cambio, continuó refiriéndose a J.F. como un hombre y, más concretamente, como un hombre homosexual. Esto se debe a que recurrió como fuente a la asociación ATC Rainbow, desde la que declararon en nombre de la familia que J.F. era un hombre homosexual. De hecho, esta aclaración fue publicada en El Diario de Valladolid tanto el 24 como el 25 de agosto, probablemente debido a que El Norte de Castilla también recurrió el 23 a la misma fuente, pero no varió la etiqueta de transexual. Por otra parte, La Razón representó a J.F. como un hombre que practicaba el travestismo tras ponerse en contacto con conocidos de la víctima.

En la fase del Juicio, las tres cabeceras que hicieron seguimiento del caso mantuvieron invariable el hecho de diversidad sexual que atribuían a J.F., si bien El Diario de Valladolid dejó de especificarlo en sus artículos. Pese a ello, se observaron dudas respecto al sexo en El Norte de Castilla, mientras que $A B C$ pasó a representarle como hombre.

\section{Discusión}

Este análisis de contenido cuantitativo tiene por objetivos analizar la representación mediática del travestismo y comprobar la aplicación de los códigos deonto- 
lógicos a través de la cobertura mediática del caso de La Eli. Para ello, se analizaron todos los artículos publicados en la prensa impresa generalista de Castilla y León sobre este suceso $(n=31)$.

A través de este procedimiento se ha comprobado que, en general, la prensa cumple con los criterios deontológicos inherentes a la cobertura mediática de sucesos y también de las identidades trans(género). Teniendo en cuenta que en las noticias sobre sucesos publican la mayor cantidad de «elementos deslegitimadores» (Billard, 2016), esto sugiere que la cobertura sobre las identidades trans(género) ha mejorado respecto a otros casos estatales (Carratalá, 2011) y también internacionales (por ejemplo, Baptista y Himmel, 2016; Barker-Plummer, 2013). Sin embargo, todavía se observa confusión a la hora de referirse tanto a su sexo como al hecho de diversidad sexual. De hecho, se han identificado dos líneas de representación paralelas, lideradas por las dos cabeceras que mayor seguimiento mediático han otorgado al caso de análisis y que han puesto al frente a redactores propios.

Por un lado, El Diario de Valladolid, la cabecera que más importancia ha otorgado al caso de la Eli, mantiene durante toda su cobertura la identidad sexual masculina de F.J. Ante la duda sobre el hecho de diversidad sexual, en un inicio opta por usar transgénero, término paraguas que integra entre otros el travestismo y la transexualidad. Más avanzado el caso, en cambio, se decanta por referirse a la orientación sexual del deseo erótico del mismo, aludiendo a su homosexualidad. De esta manera, se refiere a dos de las dimensiones que interseccionan en la vida F.J.

La segunda cabecera que más seguimiento hace del caso es El Norte de Castilla. Este comienza a representarle como un hombre que se traviste, pero al tercer día cambia a representarlo como persona transexual para, más adelante, categorizarle como mujer transexual. Al ser el que da la primicia de la muerte de F.J., $A B C$, el Diario de León, La Opinión y El Mundo (la edición nacional de El Diario de Valladolid) se adhieren a esta línea de representación a través de la información facilitada por las agencias de comunicación, que citan a El Norte de Castilla como fuente. Por tanto, la representación errónea de F.J. como una mujer en situación de transexualidad se convierte en la más habitual.

Esta desconexión entre las dos diferentes líneas de representación parece ser fruto de las fuentes utilizadas. A pesar de que el carácter noticioso de este tipo de sucesos obliga a recurrir principalmente a fuentes institucionales, el uso complementario de fuentes activistas suele mejorar la cobertura, haciéndola más completa, precisa y respetuosa (Barker-Plummer, 2013). Sin embargo, en esta ocasión las fuentes activistas ofrecen versiones diferentes respecto a la identidad sexual F.J. y su sexualidad no normativa, iniciando así dos líneas de representación temporalmente paralelas pero conceptualmente divergentes: mientras que El Diario de Valladolid se adhiere a la versión de una asociación local que habla en nombre de la familia, El Norte 
de Castilla mantiene la recomendación inicial de una organización de carácter estatal con subdelegación en Castilla y León tras tener ambas en cuenta.

También se observan indicios a través de la identidad sexual de una posible (con)fusión del travestismo con la transexualidad entre las cabeceras que eligen la línea de representación de la transexualidad. Mientras que $A B C$ cambia la identidad sexual de F.J. de mujer a hombre entre su muerte y el juicio, El Norte de Castilla opta por no especificarla, si bien en algunos de sus textos intercambia indiscriminadamente el género gramatical. Como consecuencia, se representa explícitamente una identidad sexual ambigua a la par que sus dudas implícitas respecto a la misma. Además, el que buena parte del corpus utilice los términos para referirse a los hechos de diversidad sexual como sustantivos en vez de como adjetivos deja constancia de que los medios de comunicación todavía no han interiorizado que estas facetas de la sexualidad son una dimensión más de la persona y en ningún caso un rasgo definitorio.

Esta (con)fusión entre el travestismo y la transexualidad no resulta novedosa a nivel mediático. Desde la investigación (Carratalá, 2011), las instituciones (por ejemplo, Consejo del Audiovisual de Cataluña, 2017) y el activismo (por ejemplo, El Hombre Transexual, 2006) se ha hecho hincapié en no confundir la transexualidad con el travestismo, reacción que probablemente se deba a una tendencia mediática que ha favorecido no solo la visibilidad del travestismo en detrimento de la transexualidad, sino también la representación sistemática de mujeres y hombres en situación de transexualidad como mujeres y - sobre todo- hombres que practican el travestismo. Este caso resulta paradigmático porque en esta ocasión es el travestismo el que se ha confundido con la transexualidad. Puesto que no se trata de un trabajo longitudinal, el análisis de tendencias excede los resultados de esta aproximación. Sin embargo, la notoriedad mediática que ha adquirido la transexualidad en los últimos años (Åkerlund, 2018; Billard, 2016) hace pensar en un cambio de tendencia en la (con)fusión de estas (trans)identidades.

Más allá de cuestiones relacionadas con la diversidad sexual, la evolución respecto a la forma y el contenido del seguimiento mediático responde, al menos, a dos factores más. Por un lado, a la noticiabilidad de cada una de las fases, siendo el periodo inicial de Agresión y Detención el que más atención mediática ha suscitado. Por otro, se ha identificado un proceso de despersonalización. Durante la primera fase F.J. aparece como una víctima anónima de agresión a través la mirada de fuentes institucionales, pero también desde el activismo. En el periodo de Muerte $y$ Luto, en cambio, se representa a F.J. como una persona: se le identifica y para referirse a él se utilizan con menor frecuencia que en la fase anterior términos victimizantes. En ello probablemente influya el que se dote de más espacio a fuentes activistas. Finalmente, durante la cobertura del Juicio F.J. es despersonalizado para convertirse en víctima de nuevo y pasar a un segundo plano mediante el uso exclusivo de fuentes institucionales. 


\subsection{LimitACIONES Y FORTALEZAS}

El presente trabajo tiene ciertas limitaciones, algunas de la cuales han sido parcialmente resueltas. Para empezar, la falta de una prueba de fiabilidad intercodificadores ha tratado de resolverse mediante un repaso exhaustivo de la codificación. Asimismo, la reducida cantidad de artículos que versan sobre el estudio de caso dificulta, cuando no impide, el uso adecuado de estadísticos de contraste y otras herramientas estadísticas más avanzadas. Aun así, también ha permitido un análisis más minucioso del que habría sido posible con una muestra mayor. Por último, el propio diseño como estudio de caso impide la generalización de los resultados.

Pese a ello, la combinación de variables que abordan diferentes dimensiones, desde la forma a la diversidad sexual, junto a la exhaustividad que ha posibilitado el diseño de estudio de caso, han permitido responder con éxito a objetivos planteados, generar nuevas hipótesis e interrogantes para futuras investigaciones y contribuir al conocimiento sobre la representación mediática del travestismo en diálogo con otro hecho de diversidad sexual: la transexualidad.

\section{Conclusiones}

En este estudio de caso se ha abordado de forma pormenorizada la representación mediática del travestismo. Pese a ofrecer una cobertura correcta a nivel deontológico, la prensa continúa mezclando diferentes hechos de diversidad sexual; especialmente, transexualidad y travestismo. Sin embargo, los resultados de esta investigación hacen pensar en un cambio de direccionalidad en la (con)fusión de estas (trans)identidades: de confundir la transexualidad con el travestismo, ahora parece que se confunde el travestismo con la transexualidad.

Futuros esfuerzos académicos podrían comprobar empíricamente a través de un análisis longitudinal si efectivamente existía previamente una tendencia mediática a confundir la transexualidad con el travestismo y si esta se ha invertido. Asimismo, el presente estudio de caso resulta significativo, pero no representativo. Dada la inexistencia de análisis que permitan la generalización de los resultados, también queda pendiente arrojar más luz sobre la representación mediática general del travestismo.

\section{Referencias}

Åkerlund, M. (2018). «Representations of Trans People in Swedish Newspapers». Journalism Studies, 20(9), 1319-1338. doi: 10.1080/1461670X.2018.1513816

Baptista, M. M., Y Himmel, R. (2016). “For fun”: (De) humanizing gisberta--the violence of binary gender social representation». Sexuality $\&$ Culture, 20(3), 639-656. doi: 10.1007/s12119-016-9350-5 
Barker-Plummer, B. (2013). «Fixing Gwen: News and the mediation of (trans)gender challenges». Feminist Media Studies, 13(4), 710. doi: 10.1080/14680777.2012.679289

Billard, T. (2016). "Writing in the margins: Mainstream news media representations of transgenderism». International Journal of Communication, 10, 4193-4218.

Budd, R. (1964). "Attention score: a device for measuring news 'play'». Journalism Quarterly, 41(2), 259-262.

Butler, Judith (2004). Undoing gender. Nueva York: Routledge.

Capuzza, J. C.. (2016). «Improvements still needed for transgender coverage». Newspaper Research Journal, 37(1), 82-94. doi: 10.1080/15532739.2014.946195

Carratalá, A. (2011). « ¿Smail o cristina? Desigualdad e identidad transexual en el discurso periodístico». Zer-Revista De Estudios De Comunicación, 16(31), 183-201.

Chávez, K. (2010). «Spatializing gender performativity: Ecstasy and possibilities for livable life in the tragic case of victoria arellano». Women's Studies in Communication, 33(1), 1-15. doi: 10.1080/07491401003669729

Col-legi de Periodistes de Catalunya (2016). Código deontológico Declaración de principios de la profesión periodística en Cataluña. [Fecha de consulta: 10/010/2020]. Recuperado de https://www.periodistes.cat/codi-deontologic

Consell de l'Audiovisual de Catalunya (2017). Recomendaciones sobre el tratamiento de las personas lesbianas, gays, bisexuales, transgéneros e intersexuales (LGBTI) en los medios audiovisuales. [Fecha de consulta: 10/10/2020]. Recuperado de https://www.cac.cat/ sites/default/files/2018-02/Recomanacions_LGBTI_ES.pdf

De la Serna, V. (1996). Libro de estilo de El Mundo. Madrid: Ediciones Temas de Hoy.

De Sousa, J. M. (2003). Libro de estilo Vocento. Gijón: Ediciones Trea.

El Hombre Transexual (2006). El semáforo de las palabras sobre transexualidad: Guía para el tratamiento informativo de noticias relacionadas con la transexualidad. [Fecha de consulta: 10/10/2020]. Recuperado de http://transexualia.org/wp-content/ uploads/2015/03/Apoyo_palabrassemaforo.pdf

Federación de Asociaciones de Periodistas de España (2017). Código deontológico. [Fecha de consulta: 10/10/2020]. Recuperado de http://fape.es/home/codigo-deontologico/

Federación Internacional de Periodistas (1986). Declaración de principios de la FIP sobre la conducta de los periodistas. [Fecha de consulta: 10/10/2020]. Recuperado de https:// www.ifj.org/es/quien/reglas-y-politica/declaracion-de-principios-de-la-fip-sobre-laconducta-de-los-periodistas.html

Gomis, L (1991). Teoría del periodismo: cómo se forma el presente. Barcelona: Paidós.

Grant, J., Mottet, L., Tanis, J., Harrison, J., Herman, J., Y Keisling, M. (2011). Injustice at Every Turn: A Report of the National Transgender Discrimination Survey. Washington: National Center for Transgender Equality and National Gay and Lesbian Task Force. [Fecha de consulta: 15/05/2020]. Recuperado de https://www.transequality.org/sites/default/files/docs/resources/NTDS_Report.pdf 
Krippendorff, K. (2004). Content analysis: An introduction to its methodology (2. ${ }^{a}$ edición). Thousand Oaks: Sage Publications.

Landarroitajauregi, J. R. (2000). "Términos, conceptos y reflexiones para una comprensión sexológica de la transexualidad». Anuario de sexología, 6, 79-126.

Leandro De Jesús, F., y Caldas-Coulthard, C. R. (2017). «Abjection and condemnation: Media representations of a transgender criminal in Brazil». Gender and Language, 12(3), 372-397. doi: 10.1558/genl.33299

Mackenzie, G., Y Marcel, M. (2009). "Media coverage of the murder of U.S. transwomen of color». En Cuklanz, L., Y Moorti, S. (eds.), Local violence, global media: Feminist analyses of gendered representations, pp. 79-106. New York: Peter Lang.

Mccombs, M. (2006). Estableciendo la agenda: el impacto de los medios en la opinión pública y en el conocimiento. Barcelona: Paidós.

Marini, L., Medina, P., y Rodrigo, M. (2010). «Prensa on-line y transexualidad: análisis de la cobertura periodística del caso de Thomas Beatie». Estudios sobre el mensaje periodístico, 16, 291-306.

Olveira-Araujo, R. (2019). «Trans en los media: Un estudio comparativo piloto del tratamiento de la transexualidad en la prensa generalista y los medios de comunicación digitales». Mediatika, 17, 151-172.

Parlamento de Europa (1993). Código Deontológico Europeo de la Profesión Periodística. [Fecha de consulta: 10/10/2020]. Recuperado de: http://www.asociacionprensa.org/ es/images/Codigo_Deontologico_Europeo_de_la_Profesion_Periodistica.pdf

Transgender Europe (2018). Trans day of remembrance 2018. [Fecha de consulta: 01/06/2020]. Recuperado de: https://transrespect.org/en/trans-murder-monitoring/ tmm-resources/

Unesco (1983). Código internacional de ética periodística. [Fecha de consulta: 10/10/2020]. Recuperado de: http://www.editorialdigitaltecdemonterrey.com/materialadicional/ p002/cap1/el_unesco.pdf

Velasco Malagón, T. E. (2016). «Representaciones Sociales De La Transexualidad Y De Las Personas Transexuales En España». (Tesis doctoral). Universidad Complutense de Madrid, España. Recuperado de https://eprints.ucm.es/42016/1/T38610.pdf

Vigara Tauste, A. M. (2001). Libro de estilo de ABC. Barcelona: Ariel.

Yin, R. K. (2009). Case study research: Design and methods (4. ${ }^{a}$ edición). Londres: Sage. 